\title{
Retreatment of Hepatitis C Infection With Direct-Acting Antivirals
}

\author{
Marisel Segarra-Newnham, PharmD, MPH, BCPS, BCIDP; Nathalie See, PharmD; and Gail Fox-Seaman, ARNP
}

Background: Treatment of hepatitis $\mathrm{C}$ virus (HCV) infection with direct-acting antivirals (DAAs) results in sustained virologic response (SVR) in $>90 \%$ of patients. However, some patients required retreatment with newer DAA options. Treatment was selected after consultation with a clinical pharmacy specialist.

Methods: A retrospective chart review of patients at the West Palm Beach Veterans Affairs Medical Center (WPBVAMC) in Florida retreated from January 2015 to December 2019 was conducted. Data collected included HCV genotype, previous therapy, newly prescribed medications, and treatment outcomes.

Results: Since 2015 , > 900 patients have been treated at WPBVAMC, including 22 patients who had previously failed interferon combined with DAA regimens and 46 patients who needed retreatment after failure with an all-oral therapy. This review documents the outcomes of retreatment with DAA after initial failure to achieve SVR Of 28 patients treated with a boceprevir-based regimen, 10 ended in failure. All 10 were retreated, and all achieved SVR with ledipasvir/sofosbuvir. Of 53 patients treated with a sofosbuvir-based interferon regimen, 12 failed treatment. All 12 were retreated and all achieved SVR. Thirty patients were retreated after failure with an all-oral DAA. Of 27 tested, 21 achieved SVR. All patients who failed therapy again had cirrhosis.

Conclusions: Veterans retreated with DAAs for HCV infection had a high success rate. Repeat failures of DAAs were rare, but cirrhosis seems to be common among these patients.
Marisel Segarra-Newnham is an Infectious Diseases Clinical Pharmacy Specialist; at the time of the project, Nathalie See was a

Pharmacy Resident; and Gail Fox-Seaman is a Nurse Practitioner; all at the West Palm Beach Veterans Affairs Medical Center in Florida. Nathalie See is a Post Graduate Year 2 Pharmacy Practice Resident at University of Florida Health Jacksonville.

Correspondence: Marisel Segarra-Newnham (marisel.segarra-newnham @va.gov)
A n estimated 3.5 million people in the US have chronic hepatitis $\mathrm{C}$ virus (HCV) infection, and between $10 \%$ and $20 \%$ of those developed cirrhosis over 20 to 30 years. ${ }^{1}$ There are at least 6 genotypes (GTs) of HCV, with GT1 being the most common in the US and previously one of the most difficult to treat. ${ }^{2,3}$ The goal of treatment is to achieve viral cure, called sustained virologic response (SVR) when HCV viral load remains undetectable several weeks after therapy completion. In the 2000s, pegylated interferon (pegIFN) and ribavirin (RBV) were the standard of care. ${ }^{2}$ For patients with GT1 infections, an SVR of 40 to $50 \%$ was commonly seen after 48 weeks of pegIFN/RBV regimens compared with 70 to $80 \%$ SVR for GT2 or GT3 after 24 weeks of pegIFN/RBV therapy. ${ }^{2}$ However, treatment has evolved rapidly (Table 1). ${ }^{2-17}$

In 2011, the US Food and Drug Administration (FDA) approved the protease inhibitors (PIs) boceprevir and telaprevir, which added a new class of agents with increased SVR for patients with GT1 infection; however, pegIFN and RBV were still needed for treatment. ${ }^{4}$ In addition, both PIs required multiple doses per day and strict adherence to an 8-hour schedule. ${ }^{4}$ Boceprevir required treatment with RBV and pegIFN for 48 weeks unless futility rule was met at 24 weeks of treatment (ie, viral load still detectable). ${ }^{4}$ The SVR in patients with GT1 infection improved to $>65 \%$ for patients in clinical trials. ${ }^{2}$ FDA approval of the direct-acting antivirals (DAAs) sofosbuvir and simeprevir in late 2013 decreased the usual duration of therapy to only 12 weeks with improved SVR rates 12 weeks posttherapy (SVR12) to $90 \%$ or higher. ${ }^{2,6,10}$

FDA approval of ledipasvir (LDV)/sofosbuvir (SOF) in October 2014 resulted in the first interferon-free all-oral regimen indicated for HCV GT1 infection. ${ }^{11}$ In December 2014, FDA approved a combination of paritaprevir, ritonavir, ombitasvir, and dasabuvir (PrOD). ${ }^{12}$ In 2015 GT-specific approvals were issued for daclastavir to be used with SOV for GT1 and GT3 and a combination similar to PrOD without dasabuvir (PrO) for GT4. ${ }^{13}$ In 2016, a combination of elbasvir (ERB) and grazoprevir (GZP) was approved for GT1 and GT4. ${ }^{14}$

In 2016, a pangenotypic DAA of SOF and velpatasvir (VEL) was approved..$^{15}$ Most recently, combinations of SOF, VEL, and voxilaprevir (VOX), and glecaprevir (GLE) and pibrentasvir (PIB) were approved for patients with previous DAA treatment failures. ${ }^{7,8,16,17}$ These oral regimens avoided the significant adverse events (AEs) associated with pegIFN and RBV (eg, thrombocytopenia, depression), were expected to improve treatment adherence and shorten duration of therapy. 
The West Palm Beach Veterans Affairs has had a nurse practitioner (NP)-based HCV treatment clinic since the late 1990s. When PIs became available, a CPS started reviewing patient electronic health records (EHRs) and monitored response to therapy along with the NP to ensure discontinuation of therapy if futility criteria were met. ${ }^{7}$ Our unpublished experience showed SVR $>60 \%$ with both boceprevir and SOF regimens and $>90 \%$ with oral DAA regimens.

This review will provide the SVR rates for patients that needed retreatment for $\mathrm{HCV}$ infection since 2015 until December 2019. We treated all willing patients, beginning with the patients who had experienced failures with previous regimens. Patients first received education on $\mathrm{HCV}$ infection and treatment options in a group class then they were seen by the NP individually for specific education on treatment. The CPS reviewed the patient's medical record to assess for appropriate therapy, possible drug-drug interactions and contraindications to therapy. In addition, patient outcomes (eg, viral load, AEs) were documented by the CPS in collaboration with the NP throughout treatment until viral load for SVR evaluation was obtained.

\section{METHODS}

A retrospective EHR review of patients retreated from January 2015 to December 2019 was conducted. Data collected included age, sex, HCV GT, previous therapy, new medications prescribed, creatinine clearance, and achievement of SVR12. This retrospective review was approved by the facility's scientific advisory committee as part of performance improvement efforts. Descriptive statistics are provided.

\section{RESULTS \\ Boceprevir}

We treated 31 patients with boceprevir of which 3 met futility rule and 28 completed therapy. Eighteen of 28 responded (64\%) to the treatment. The 10 patients who failed treatment were retreated with LDV/SOF, and all achieved SVR.

\section{Sofosbuvir}

A total of 53 patients were treated with SOF, RBV, and pegIFN for 12 weeks. Forty-one
TABLE 1 Treatment Milestones for Hepatitis C Infection

\begin{tabular}{|c|c|}
\hline Years & Treatment Milestones \\
\hline 2000s & Interferon-based therapy with ribavirin for 24 to 48 wk depending on GT \\
\hline 2011 & $\begin{array}{l}\text { Boceprevir and telaprevir (protease inhibitors) for GT1 for } 48 \text { wk combined } \\
\text { with interferon and ribavirin }\end{array}$ \\
\hline 2013 & $\begin{array}{l}\text { SOF and simeprevir for GT1; treatment for } 12 \text { wk combined with interferon } \\
\text { and ribavirin }\end{array}$ \\
\hline 2014 & $\begin{array}{l}\text { SOF/ledipasvir and PrOD; all-oral for } 8 \text { wk (SOF/ledipasvir) or } 12 \text { wk; some } \\
\text { patients required ribavirin }\end{array}$ \\
\hline 2015 & Daclatasvir (GT1 and GT3) and PrO (GT4) ; all-oral treatment for 12 wk \\
\hline 2016 & $\begin{array}{l}\text { ERB/GZP for GT1 and GT4; SOF/VEL for all genotypes; all oral treatment } \\
\text { for } 12 \mathrm{wk}\end{array}$ \\
\hline 2017 & $\begin{array}{l}\text { GLE/PIB and SOF/VELNOX; all-oral treatment, options for previously } \\
\text { treated patients, treatment } 16 \text { wk (GLE/PIB) or } 12 \text { wk; GLE/PIB can be } \\
\text { used for } 8 \text { wk in treatment-naïve patients }\end{array}$ \\
\hline
\end{tabular}

Abbreviations: ERB, elbasvir; GLE, glecaprevir; GT, genotype; GZP, grazoprevir; PIB, pibrentasvir; PrO, paritaprevir, ritonavir, and ombitasvir; PrOD, paritaprevir, ritonavir, ombitasvir, and dasabuvir; SOF, sofosbuvir; VEL, velpatasvir; VOX, voxilaprevir.

TABLE 2 SVR Following Failed Treatment With Sofosbuvir, Ribavirin, and Pegylated Interferon $(n=12)$

\begin{tabular}{lll} 
Genotypes & Medications Used & SVR Achieved, No. \\
1 & Ledipasvir/sofosbuvir & 5 \\
& Sofosbuvir/velpatasvir & 1 \\
& Sofosbuvir/velpatasvir/voxilaprevir & 1 \\
\hline 2 & Sofosbuvir/velpatasvir & 3 \\
\hline 3 & Sofosbuvir/velpatasvir & 2
\end{tabular}

Abbreviation: SVR, sustained virologic response.

achieved SVR (77\%). Of the 12 who failed therapy, all have been retreated and achieved SVR (Table 2).

\section{Interferon-Free DAA Oral Regimens}

More than 900 patients have been treated with interferon-free regimens since 2015 and outcomes were documented for $>800 \mathrm{pa}$ tients. The SVR rates by GT were as follows: GT1 639 of 676 (95\%); GT2 76 of 79 (96\%); GT3 40 of 48 (83\%); and GT4 6 of 6 (100\%). Eighty-four percent of patients had GTl infection. The median age of patient was 62 years, $72 \%$ were treatment naive, and $35 \%$ having cirrhosis (based on liver biopsy or FIB4 score). ${ }^{18}$

Of 48 treatment failures, 30 patients were retreated; the rest of the patients were lost to follow-up $(n=9)$ or unable to 
TABLE 3 SVR After Ledipasvir/SOF Failure $(n=22)$

\begin{tabular}{|c|c|c|c|c|}
\hline GT & Treatment Details & No. & SVR, No. (\%) & Treatment Failures \\
\hline 1 & $\begin{array}{l}\text { 8-wk treatment regimen } \\
\text { SOF/NEL } \\
\text { ERB/GZP } \\
\text { SOF/VEL/VOX }\end{array}$ & $\begin{array}{l}10 \\
2 \\
1 \\
7\end{array}$ & $\begin{array}{c}7(88) \\
1 \\
1 \\
5\end{array}$ & $\begin{array}{l}1 \\
1^{\mathrm{a}} \\
-- \\
1^{\mathrm{b}}\end{array}$ \\
\hline 1 & $\begin{array}{l}\text { 12-wk treatment regimen } \\
\text { SOF/VEL } \\
\text { SOF/NEL/VOX } \\
\text { GLE/PIB }^{\mathrm{b}}\end{array}$ & $\begin{array}{l}9 \\
5 \\
2 \\
2\end{array}$ & $\begin{array}{l}4(57) \\
3 \\
1 \\
--\end{array}$ & $\begin{array}{l}3 \\
2 \\
1 \\
--\end{array}$ \\
\hline 3 & SOF/VEL & 3 & $1(33)$ & 2 \\
\hline
\end{tabular}

Abbreviations: ERB, elbasvir; GLE, glecaprevir; GT, genotype; GZP, grazoprevir; PIB, pibrentasvir; SOF, sofosbuvir; SVR, sustained virologic response; VEL, velpatasvir; VOX, voxilaprevir.

aSVR achieved with SOF/VEL/VOX

bSVR pending.
TABLE 4 Retreatment After ERB/GZP Failure Genotype $1(n=5)$

\begin{tabular}{lcc} 
Treatments & SVR & Total, No. \\
\hline SOF/VEL & 2 & 2 \\
SOF/VEL/NOX & 2 & 3
\end{tabular}

Abbreviations: ERB, elbasvir; GZP, grazoprevir; SOF, sofosbuvir; VEL, velpatasvir; VOX, voxilaprevir; SVR, sustained virologic response.

a1 lost to follow-up.

shown to improve fibrosis, portal hypertension, splenomegaly and cirrhosis, and reduce the risk of hepatocellular carcinoma by $70 \%$ and liver-related mortality by $90 \% .{ }^{19-21 /}$

Patients who failed therapy primarily had GT1 - the most prevalent GT treated. A higher prevalence of GT1 is expected since it is the most common GT in the US. ${ }^{6}$ However, disease progression occurs more rapidly in those with GT3 and is more difficult to treat. ${ }^{22}$ The overall response rate was lower with this GT (83\%) in this report, with only 1 of 3 patients retreated achieving an SVR.

Similar results are documented in retreatment trials. ${ }^{23}$ In the POLARIS-1 trial, treatment with SOF/VEL/VOX resulted in an overall response rate of $96 \%$ but only $91 \%$ for patients with GT3, compared with 95 to $100 \%$ for GTs 1,2 , or $4 .{ }^{23}$ In the current report, only 1 patient (GT1) failed retreatment with SOF/VEL/VOX. At this time, there are no clear treatment options for this patient. However, patients who fail GLE/PIB (none so far in the current report) may be able to receive SOF/VEL/VOX. ${ }^{24}$ In a small study, 29 of 31 patients achieved SVR with SOF/ VEL/VOX after GLE/PIB failure (12 of 13 GT1 and 17 of 18 GT3). ${ }^{24}$

\section{Limitations}

This review was an observational, nonrandomized design, and only 1 medical center was involved. These results may not be applicable to other patient populations without a clinic set up with routine follow-ups to encourage adherence and completion of therapy.

\section{CONCLUSIONS}

Treatment of HCV infection has improved significantly over the past 10 years. Use of DAAs results in SVR for > 90\% of patients, especially if the disease had not progressed to cirrhosis. Failure after retreatment for $\mathrm{HCV}$ 
infection was rare as well. Given that cirrhosis seems to increase the chance of treatment failure, it is imperative to identify candidates for treatment before the infection has progressed to cirrhosis. Patients infected with GT3 in particular should be more aggressively identified and treated.

\section{Acknowledgments}

The authors thank Nick P. Becky, PharmD, for his contributions to the identification of patients needing treatment for their HCV infection and review of initial manuscript information.

\section{Author disclosures}

The authors report no actual or potential conflicts of interest with regard to this article.

\section{Disclaimer}

The opinions expressed herein are those of the authors and do not necessarily reflect those of Federal Practitioner, Frontline Medical Communications Inc., the US Government, or any of its agencies. This article may discuss unlabeled or investigational use of certain drugs. Please review the complete prescribing information for specific drugs or drug combinations-including indications, contraindications, warnings, and adverse effects-before administering pharmacologic therapy to patients.

\section{References}

1. Centers for Disease Control and Prevention. Viral hepatitis: hepatitis C information. https://www.cdc.gov/hepatitis /hcv/index.htm. Reviewed April 14, 2020. Accessed June 16, 2020.

2. American Association for the Study of Liver Disease, Infectious Diseases Society of America. HCV Guidance: Recommendations for Testing, Managing, and Treating Hepatitis C. https://www.hcvguidelines.org. Accessed June 16, 2020.

3. Lingala S, Ghany MG. Natural history of hepatitis C. Gastroenterol Clin N Am. 2015;44(4):717-734. doi:10.1016/j.gtc.2015.07.003

4. Foote BS, Spooner LM, Belliveau PP. Boceprevir: a protease inhibitor for the treatment of chronic hepatitis C. Ann Pharmacother. 2011;45(9):1085-1093. doi:10.1345/aph.1P744

5. Kayali Z, Schmidt WN. Finally sofosbuvir: an oral anti-HCV drug with wide performance capability. Pharmgenomics Pers Med. 2014:7:387-398. doi:10.2147/PGPM.S52629

6. Falade-Nwulis O, Suarez-Cuervo C, Nelson DR, Fried MW, Segal JB, Sulkowski MS. Oral direct-acting agent therapy for hepatitis $\mathrm{C}$ virus infection: a systematic review. Ann Intern Med. 2017;166(9):637-648. doi:10.7326/M16-2575

7. Carrion AF, Martin P. Glecaprevir + pibrentasvir for treatment of hepatitis C. Expert Opinion Pharmacother.
2018;19(4):413-419. doi:10.1080/14656566.2018.1444030

8. Chahine EB, Kelley D, Childs-Kean LM. Sofosbuvir/velpatasvir/voxilaprevir: a pan-genotypic direct-acting antiviral combination for hepatitis C. Ann Pharmacother. 2018;52(4):352-363. doi:10.1177/1060028017741508

9. Lagasca AM, Kan VL. Hepatitis C treatment at a Veteran Affairs medical center after the availability of directacting agents: things are looking up. Clin Infect Dis. 2015:61(8):1347-1349. doi:10.1093/cid/civ573

10. Sovaldi (sofosbuvir) [package insert]. Foster City, CA: Gilead Sciences Inc; 2017.

11. Harvoni (ledipasvir and sofosbuvir) [package insert]. Foster City, CA: Gilead Sciences Inc; 2017.

12. Viekira Pak (ombitasvir, paritaprevir and ritonavir; dasabuvir) [package insert]. North Chicago, IL: AbbVie Inc; 2018.

13. Technivie (ombitasvir, paritaprevir and ritonavir) [package insert]. North Chicago, IL: AbbVie Inc; 2018.

14. Zepatier (elbasvir and grazoprevir) [package insert]. Whitehouse Station, NJ: Merck \& Co Inc; 2018.

15. Epclusa (sofosbuvir and velpatasvir) [package insert]. Foster City, CA: Gilead Sciences Inc; 2017.

16. Mavyret (glecaprevir and pibrentasvir) [package insert]. North Chicago, IL: AbbVie Inc; 2019.

17. Vosevi (sofosbuvir, velpatasvir and voxilaprevir) [package insert]. Foster City, CA: Gilead Sciences Inc; 2017.

18. Vallet-Pichard A, Mallet V, Nalpas V, et al. FIB-4: an inexpensive and accurate marker of fibrosis in HCV infection. Comparison with liver biopsy and FibroTest. Hepatology. 2017;46(1):32-36. doi:10.1002/hep.21669

19. Morgan RL, Baack B, Smith BD, Yartel A, Pitasi M, Falck-Ytter Y. Eradication of hepatitis $C$ virus infection and the development of hepatocellular carcinoma: a meta-analysis of observational studies. Ann Intern Med. 2013;158(5, pt 1):329-337. doi:10.7326/0003-4819-158-5-201303050-00005

20. van der Meer AJ, Veldt BJ, Feld JJ, et al. Association between sustained virological response and all-cause mortality among patients with chronic hepatitis $\mathrm{C}$ and advanced hepatic fibrosis. JAMA. 2012;308(24):2584-2593. doi:10.1001/jama.2012.144878

21. Veldt BJ, Heathcote EJ, Wedemeyer $H$, et al. Sustained virologic response and clinical outcomes in patients with chronic hepatitis $\mathrm{C}$ and advanced fibrosis. Ann Intern Med. 2007;147(10):677-684. doi:10.7326/0003-4819-147-10-200711200-00003

22. Chen A, Patel K, Naggie S. Genotype 3 infection: the last stand of hepatitis C virus. Drugs. 2017;77(2):131-144. doi:10.1007/s40265-016-0685-x

23. Bourlière M, Gordon SC, Flamm SL, et al; POLARIS-1 and POLARIS-4 Investigators. Sofosbuvir, velpatasvir and voxilaprevir for previously treated HCV infection. N Engl J Med. 2017;376(22):2136-2146. doi:10.1056/NEJMoa1613512

24. Pearlman B, Perrys M, Hinds A. Sofosbuvir/velpatasvir/voxilaprevir for previous treatment failures with glecaprevir/pibrentasvir in chronic hepatitis $\mathrm{C}$ infection. Am J Gastroenterol. 2019;114(9):1550-1552. doi:10.14309/ajg.0000000000000248 